\title{
La Prevalenza del Dolore Cronico Non Oncologico nell'Adulto e Patologie Associate: una revisione narrativa della letteratura
}

\author{
Etiology and prevalence of chronic pain in adults a Narrative Review
}

Roberto Latina $^{1} \quad$ Julita Sansoni ${ }^{2}$ Daniela D'Angelo ${ }^{3} \quad$ Ettore Di Biagio $^{4}$

Maria Grazia De Marinis 5 Gianfranco Tarsitani 6

\section{RIASSUNTO}

Il dolore cronico non oncologico rappresenta un problema sanitario sottostimato dal punto di vista epidemiologico ed in termini di salute pubblica. Esso è una malattia in senso stretto, e rappresenta una delle maggiori motivazioni per cui i soggetti si rivolgono ai servizi sanitari. La grandezza del fenomeno si esprime in termini di sofferenza umana e di costi sociali. Lobiettivo di questa revisione è quello di identificare le cause e la prevalenza del dolore cronico non oncologico nei soggetti adulti.

E' stata realizzata una revisione della letteratura dal 1998 al 2012 utilizzando le emeroteche virtuali partendo dalle banche dati (Pub-Med, CINHAL, Cochrane). È stata effettuata una revisione narrativa degli articoli ottenuti. Sono stati esclusi articoli riguardanti cefalee, fasce di età pediatriche e geriatriche, oncologici e articoli specifici per patologia. Gli studi ottenuti sono stati classificati per anno, autore campione, metodi, fasce di età e definizione di dolore. Sono stati selezionati 7 articoli. Questi studi epidemiologici, realizzati in diverse parti del mondo, riportano un tasso di prevalenza relativo al dolore cronico tra il 16-53\%. Emerge una elevata eterogeneità di risultati relativi alle diagnosi e metodi. Seppure limitato il numero degli articoli, emerge una elevata complessità del fenomeno.

Parole chiave: Dolore Cronico, Prevalenza, Epidemiologia

ABSTRACT

The chronic nonmalignant pain is an underestimated epidemiologic health problem. It is a disease in its own right. It is one of the major reasons because patients use health service. The magnitude of chronic pain is in terms of human suffering and costs to society. The aim of this review is to identify the diagnosis and the prevalence of nonmalignant chronic pain in the adults.

We have done a review of the literature from 1998 to 2012 using the virtual newspaper libraries starting from data bases (Pub-Med, CINAHL, Cochrane). We have made a narrative review of the articles obtained. Excluding topics of headache, pain for pediatric and geriatric groups, cancer pain and disease-specific items. Studies were classified for year, author sample, methods, age groups and definition of pain.

We have obtained 7 articles. These epidemiological studies conducted in different part of the world, reported prevalence rates of chronic pain ranging from 16-53\%. They shows a high heterogeneity of results concerning diagnosis and methods. Although limited the number of articles, show the high complexity of the phenomenon.

Key Words: Chronic Pain, Prevalence, Epidemiology.

INTRODUZIONE

$I^{\prime}$ dolore cronico rappresenta un problema per la salute pubblica ancora oggi sottostimato, che genera un peggioramento della qualità di vita dei soggetti che ne sono affetti ed un conseguente uso dei servizi sanitari

1 Dottorando di Ricerca in Scienze di Sanità Pubblica e Microbiologia, Sapienza Università di Roma, Piazzale A. Moro 5, 00189 Roma, Italy. Corrispondenza: roblatina@gmail.com, roberto.latina@uniroma1.it

2 Professore Ordinario di Scienze Infermieristiche. Università di Primoskem (Slovenia). Professore Associato Med 45 Sapienza Università di Roma Piazzale A. Moro 5, 00189 Roma, Italy

3 Dottorando di Ricerca in Scienze Infermieristiche, Università Tor Vergata, Via Montpellier 1, 00133 Roma, Italy.

4 Medico Odontoiatra, Piazzale Medaglie d'Oro 34, 00136 Roma, Italy

5 Professore Associato di Scienze Infermieristiche, Presidente CdL in Infermieristica, Università Campus Bio-Medico, Via Alvaro del Portillo 21, 00128 Roma, Italy

6 Professore Ordinario di Igiene, Sapienza Università di Roma, Piazzale A. Moro 5, 00189 Roma, Italy
(EFIC, 2011; Abu-Saad, 2010; Blyth et al., 2001; Breivik et al., 2006; Widerström-Noga, 2009). Secondo la International Association of Study of Pain (1994) e la American Pain Society (2008), il dolore cronico rappresenta una esperienza complessa e multidimensionale che coinvolge fattori fisici, psicologici, emozionali, sociali (Blyth et al., 2004; Eriksen et al., 2004) che si protrae per almeno 3 mesi e più (IASP, 1994). Il dolore cronico è considerato un grave problema sanitario non solo in termini di sofferenza umana, di morbilità elevata e disabilità (Sullivan et al., 1991; Gallagher, 1999), ma anche in termini di implicazioni economiche enormi per la società (Siøgren et al., 2009), in quanto può incrementare il tasso di assenteismo lavorativo e ultimamente, addirittura, può divenire causa della perdita di lavoro. Infatti è opportuno non sottovalu- 
tare il sottile effetto che il dolore cronico può avere sulle capacità di lavorare in maniera efficace (Joint Commission International, 2010).

In letteratura la prevalenza del dolore cronico è descritta con ampi range di risultati, derivanti per lo più dall'utilizzo di differenti interpretazioni del caso e definizione di dolore cronico, dalle diverse metodologie di studio e dagli innumerevoli strumenti utilizzati (Eriksen et al., 2003). La semplice scelta dei parametri temporali diversi nella definizione di dolore cronico fornisce un diverso raggio di risultati in termini di prevalenza. Inoltre la International Classification of Disease vs 10 (ICD-10) non prevede a tutt'oggi nelle versioni più aggiornate, diagnosi specifiche per il dolore cronico, considerandolo pertanto solo come la sommatoria di problemi somatici che hanno per risultato il sintomo il dolore. Questa erronea interpretazione contrasta con una visione moderna del dolore cronico come malattia in se stessa (Niv \& Devor, 2004; Varrassi et al., 2011) Le stime epidemiologiche dei problemi di salute sulla popolazione sono necessarie al fine di identificare le cause e le possibilità di intervento, per orientare le politiche in termini di programmazione sanitaria a garanzia di una offerta assistenziale proporzionale ed efficace alle necessità reali dei cittadini. Inoltre possono orientare l'utilizzo dei budget per priorità sanitarie (Bekkinering et al., 2011).

Sebbene molti Paesi abbiano sostenuto programmi di ricerca sulla stima della diffusione del dolore cronico benigno sulla popolazione generale, esigui invece sono gli studi in Italia, dove il fenomeno è pressoché sconosciuto.

L'obiettivo di questa revisione narrativa è quella di descrivere gli studi presenti in letteratura che oltre a stimare il dolore cronico non oncologico,siano in grado di identificare e descrivere le condizioni cliniche che lo caratterizzano.

\section{METODI}

La ricerca della letteratura è stata realizzata per il periodo 1998-2012, usando le parole chiave Epidemiology, Prevalence, Chronic Pain, Nonmalignant. È stato usato l'operatore booleano AND per limitare il campo di ricerca, e utilizzate le seguenti query": ((chronic pain[Title/Abstract]) AND epidemiology[Title/Abstract]) AND prevalence[Title/Abstract]”; ((chronic pain[Title/Abstract]) AND prevalence[Title/Abstract]) AND "epidemiology"[Title/Abstract] sui database PubMed e CINAHL, Cochrane. Sono stati inclusi tutti gli studi epidemiologici nazionali ed internazionali, tenendo in considerazione le fasce di età potenzialmente abili al lavoro (14-70 anni), affetti da dolore cronico non oncologico da almeno 3-6 mesi, pubblicati in lingua inglese o italiana. Sono stati esclusi tutti gli studi relativi a studi epidemiologici che facevano riferimento a monopatologie (lombosciatalgia, osteoartrosi, Herpes Zooster ect..), a fasce di età pediatriche e geriatriche, a patologie oncologiche, a cefalee/emicrania, in quanto seguono un filone epidemiologico distinto. Gli articoli ottenuti sono stati analizzati in diversi step (Figura 1), quindi classificati per anno, autore, rivista, titolo articolo, Paese, dato di prevalenza, fasce di età, definizione caso di dolore cronico, dimensione del campione, campionamento, metodologia di indagine. E stata quindi realizzata una revisione narrativa. La metodologia snowball è stata realizzata sugli articoli eleggibili ottenuti al fine di ampliare la ricercare (Figura 1).

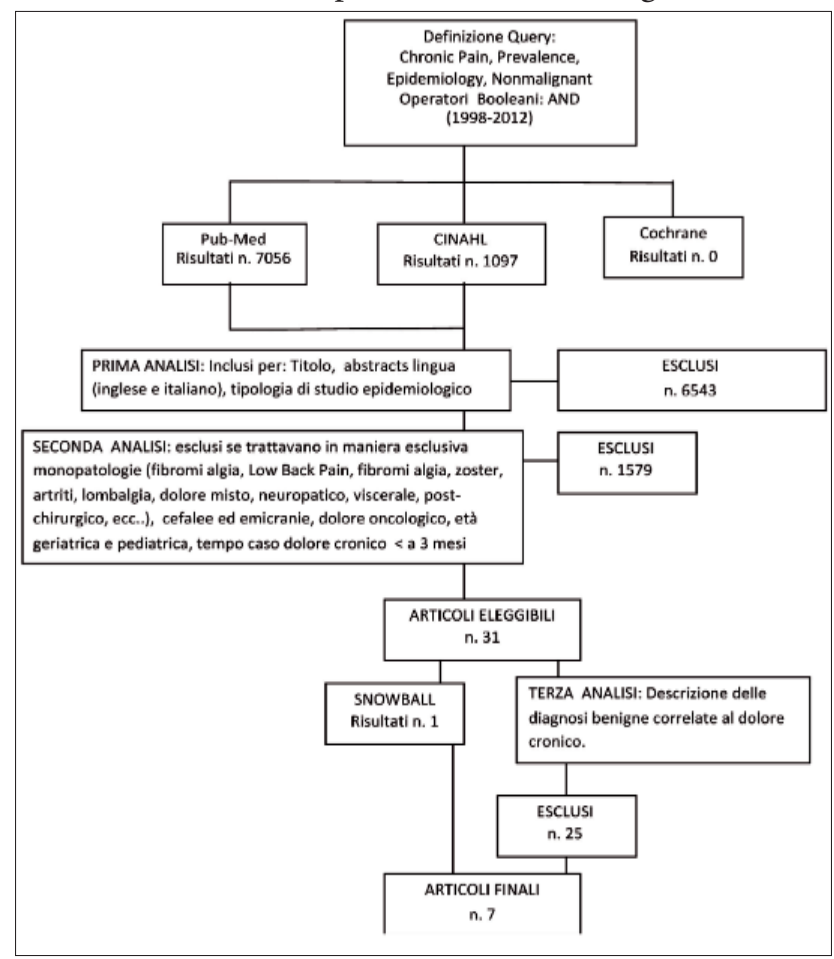

Figura 1: Selezione articoli

\section{RISULTATI}

Dopo i tre step di valutazioni realizzate sugli articoli, come descritto in Figura 1, sono stati ottenuti 31 articoli eleggibili. Questi ultimi, al fine di soddisfare i criteri di ricerca in merito all'identificazione delle diagnosi cliniche relative al dolore cronico benigno, sono stati ottenuti 7 articoli finali(Tabella 1). L'analisi degli articoli si è concentrata su: Koleva et al., 2005, Siøgren et. al., 2009, Malilis-Gagnon et al., 2007, Eriksen et al 2003, Breivik et al., 2006, Rustøen et al., 2004, Azevedo et al., 2012.

a) Definizione di caso dolore cronico benigno e criteri di inclusione della popolazione studiata

La definizione di caso "dolore cronico" rappresenta il punto di partenza per includere i soggetti nello studio epidemiologico al fine da includerlo nello stesso. Gli 


\begin{tabular}{|c|c|c|c|c|c|c|c|}
\hline Anno & Autori & $\begin{array}{l}\text { Definizione } \\
\text { Dolore }\end{array}$ & Prevalenza & Campione & Età & Strumenti usati & $\begin{array}{l}\text { Metodologia } \\
\text { di indagine }\end{array}$ \\
\hline \multirow{2}{*}{2003} & \multirow{2}{*}{ Eriksen et al., } & \multirow{2}{*}{$\geq 6$ mesi } & $21 \% \mathrm{~F}$ & \multirow{2}{*}{10066} & \multirow{2}{*}{$>16$} & \multirow{2}{*}{ SF 36} & \multirow{2}{*}{ Intervista faccia-faccia } \\
\hline & & & $16-19 \% M$ & & & & \\
\hline 2004 & Rustoen, et al., & $>3$ mesi & $24.4 \%$ & 1912 & $19-81$ & $\begin{array}{c}\text { Numerating Rating Scale, } \\
\text { altro }\end{array}$ & Mail \\
\hline 2005 & Koleva et al., & $>3$ mesi & $52.80 \%$ & 1432 & $\begin{array}{l}>14, \\
\text { adulti }\end{array}$ & Non chiaro & $\begin{array}{c}\text { Consultazione cartelle } \\
\text { del GP* }\end{array}$ \\
\hline 2006 & Breivik et al., & $\geq 6$ mesi & $19 \%$ & 4839 & $18-81+$ & $\begin{array}{c}2 \text { questionari, Numerating } \\
\text { Rating Scale }\end{array}$ & $\begin{array}{c}\text { Intervista telefonica } \\
\text { assistita }\end{array}$ \\
\hline 2007 & Mailis-Gagnon & $>3,>6$ mesi & $\begin{array}{c}\text { non dato } \\
\text { complessivo distinto } \\
\text { per gruppi di patologie }\end{array}$ & 1242 & $\begin{array}{l}\text { Tutte le } \\
\text { età }\end{array}$ & $\begin{array}{c}\text { McGill Pain } \\
\text { DSM-IV-TR Classification } \\
\text { Questionnaire Short } \\
\text { Form, altro }\end{array}$ & $\begin{array}{l}\text { Nuovi pazienti inseriti nel } \\
\text { programma di gestione del } \\
\text { dolore }\end{array}$ \\
\hline 2009 & Siøgren et al., & $>6$ mesi & $20.2 \%$ & 5292 & $16-65$ & $\begin{array}{c}\text { SF-36, ICD-10, } \\
\text { International Standard } \\
\text { Classification of Education }\end{array}$ & Intervista personale \\
\hline 2012 & Azevedo et al., & $\geq 3$ mesi & $36.7 \%$ & 5094 & $18-75+$ & $\begin{array}{l}\text { Brief Pain Inventory, } \\
\text { Pain Disability Index }\end{array}$ & Intervista telefonica assistita \\
\hline
\end{tabular}

.*GP: General Practitioner (Medico di Medicina Generale)

Tabella 1: Studi selezionati

studi descritti in Tabella 1 definiscono il caso dolore cronico come una continua esperienza che si protrae o che ha una durata di almeno 3 mesi (Koleva et al., 2005; Rustøen et al., 2004; Azevedo et al., 2012) oppure, come descritto dai rimanenti studi, di almeno 6 mesi e più (Eriksen et al 2003; Breivik et al., 2006; Malilis-Gagnon et al., 2007; Siøgren et. al., 2009). Nello studio condotto da Rustøen et al. (2004), il questionario prevedeva la seguente domande: "Da quanto tempo ha dolore ?" Coloro che rispondevano con una durata superiore a 3 mesi venivano inseriti nello studio. Nello studio di Koleva et al., (2005) si fa specifico riferimento alla definizione di dolore cronico proposto della IASP (2003). Lo stesso studio esclude patologie maligne. Nello studio condotto da Malilis-Gagnon et al.,(2007) il caso dolore cronico fa riferimento al Diagnostic and Statistical Manual of Mental Disorder, Fourth Edition-text revision (DSM -IV-TR, 2000). Negli studi Malilis-Gagnon et al., (2007) Koleva et al.,(2005), Rustøen et al., (2004), non è fatto riferimento esplicito a dolore cronico benigno e non è chiara la modalità di inclusione dei soggetti. Nello studio di Eriksen et al (2003) ai candidati veniva chiesto come criterio di inclusione: " $E$ ' affetto da dolore da 6 mesi o oltre?". In caso non affermativo venivano inseriti nel gruppo dei controlli; sono stati esclusi i soggetti affetti da patologia maligna. Nello di Siøgren et. al. (2009) sono stati usati gli stessi criteri e messi a confronto entrambi i risultati (Eriksen et al 2003). Nello studio multicentrico di Breivick et al., (2006) sono stati considerati soggetti affetti da dolore cronico coloro che soffrivano di dolore da almeno 6 mesi, e non è fatto riferimento esplicito al dolore cronico benigno.

Inoltre lo studio di Azevedo et al., (2012), fa riferimento a quanto descritto dalla tassonomia della IASP (1994), considera 3 mesi il passaggio dal dolore acuto a cronico, per quanto criticato dallo stesso e autorevole Bonica.JJ.

\section{b) Campione e metodologia di indagine.}

Tutti gli studi sottoposti a revisione narrativa sono caratterizzati da una variabile grandezza campionaria e metodologia di indagine. Infatti esse prevedono interviste face to face, telefoniche e analisi della documentazione sanitaria (Tabella 1). I soggetti inclusi nello studio condotto da Malilis-Gagnon et al., (2007) si tratta di pazienti inseriti nel Comprensive Pain Program (CPP), di durata triennale (dal 2001 al 2004), all'interno di del Western Hospital di Toronto; essi sono nuovi pazienti istituzionalizzati osservati nel corso di un triennio di studio. Nello studio di Koleva et al., (2005) l'indagine si è svolta con la collaborazione di 89 Medici di Medicina Generale (o General Practitioner nella lingua anglosassone), tramite la consultazione della documentazione sanitaria; lo studio multicentrico ha coinvolto diverse Regioni del centro, nord e sud della nazione italiana. Nello studio di Siøgren et. al., (2009) gli autori hanno comparato i dati di prevalenza con uno studio precedente (Eriksen et al, 2003). Ė stato realizzato un campionamento stratificato per regione, utilizzando l' intervista. Nello studio condotto da Breivick et al., (2006) è stato utilizzato un campione standard per ogni città, in due step (campione di 2000 persone). Lo studio è stato realizzato mediante una intervista telefonica, così come lo studio di Rustøen et al., (2004). Lo studio recente di Azevedo et al., (2012) ha identificato i soggetti in una prima fase telefonicamente, e mediante 3 steps successivi, ha selezionato in maniera randomizzata i partecipanti residenti.

Il target di studio dagli studi selezionati è popolazione generale (Azevedo et al., 2012; Rustøen et al, 2004; Breivick et al., 2006; Eriksen et al., 2003; Siøgren et. al., 2009), denominatore comune necessario al fine della determinazione dalla prevalenza. Altre ricerche sono state realizzate in collaborazione con i medici di famiglia, altri 


\begin{tabular}{|c|c|c|c|c|c|c|c|}
\hline AUTORI & $\begin{array}{l}\text { Rustøen } \\
\text { et al., } 2004\end{array}$ & $\begin{array}{c}\text { Mailis-Gagnon et } \\
\text { al., } 2007\end{array}$ & $\begin{array}{l}\text { Breivik et al, } \\
2006\end{array}$ & $\begin{array}{c}\text { Koleva } \\
\text { et al., } 2004\end{array}$ & $\begin{array}{l}\text { Eriksen } \\
\text { et al., } 2003\end{array}$ & $\begin{array}{c}\text { Siøgren et al } \\
2009\end{array}$ & $\begin{array}{l}\text { Azevedo et al } \\
2012\end{array}$ \\
\hline $\begin{array}{c}\text { DIAGNOSI CLINICHE } \\
\text { O SINTOMI }\end{array}$ & $\mathrm{P}(\%)$ & $\mathrm{P}(\%)$ & $\mathrm{P}(\%)$ & $\mathrm{P}(\%)$ & $\mathrm{P}(\%)$ & $\mathrm{P}(\%)$ & $P(\%)$ \\
\hline Fibromialgia & 8 & & & & & & 1 \\
\hline Artrite reumatoide & 9 & & 8 & & & & 5 \\
\hline $\begin{array}{l}\text { Problemi muscolo } \\
\text {-scheletrici }\end{array}$ & 24 & 25.2 & $\begin{array}{l}4 \text { danno } \\
\text { cartilagineo }\end{array}$ & $\begin{array}{c}6.1 \text { mialgia } \\
\text { 3.3 ligamenti e } \\
\text { muscoli }\end{array}$ & 57 & 668 & $\begin{array}{l}2 \text { Ginocchia e } \\
\text { spalla }\end{array}$ \\
\hline Osteoartriti & 19 & $10: 8$ & $\begin{array}{l}34 \text { Comprese } \\
\text { Artriti }\end{array}$ & & & & $\begin{array}{c}47 \text { Incluse osteo- } \\
\text { artrosi }\end{array}$ \\
\hline Osteoporosi & 4 & & & & & 392 & 15 \\
\hline Malattie gastrointestinali & 16 & & & & 47 & & $\begin{array}{c}1 \text { Crohn, Colite } \\
\text { ulcerosa }\end{array}$ \\
\hline Danno nervoso & & & 4 & & & & \\
\hline Colpo di frusta & & & 4 & & & & \\
\hline $\begin{array}{c}\text { Artropatie e disordini } \\
\text { connessi }\end{array}$ & & 6.7 spalla & & $23: 2$ & & & \\
\hline Dorsopatie & & & & $16: 5$ & & & \\
\hline $\begin{array}{c}\text { Reumatismi ad esclusione } \\
\text { infortuni lombari ed effetti } \\
\text { avversi }\end{array}$ & & & & $13: 1$ & & & \\
\hline Trauma/infortuni & & & $12+6$ fratture & $7: 6$ & 45 & 573 & 9,8 solo fratture \\
\hline Malattie endocrine & 5 diabete & & & & 40 & 301 & \\
\hline $\begin{array}{l}\text { Malattie sistema } \\
\text { circolatorio }\end{array}$ & $\begin{array}{l}7 \text { angina } \\
3 \text { IMA }\end{array}$ & $\begin{array}{l}1 \text { ischemico- } \\
\text { vascolare }\end{array}$ & & & $\begin{array}{c}38 \\
24 \text { sangue }\end{array}$ & 34 & 7 vascolari \\
\hline $\begin{array}{c}\text { Malattie del sistema nervo- } \\
\text { so }\end{array}$ & & & & & 32 & 378 & \\
\hline $\begin{array}{c}\text { Sistema respiratorio e altre } \\
\text { malattie della cute e sotto- } \\
\text { cute }\end{array}$ & 11 asma & $\begin{array}{l}0.7 \text { cute e } \\
\text { infezioni- } \\
\text { giunture }\end{array}$ & & $6: 7$ & $\begin{array}{c}32 \text { respiratori } \\
24 \text { cute e sotto- } \\
\text { cute }\end{array}$ & $\begin{array}{l}29.7+37.7 \text { solo } \\
\text { cute e sottocute }\end{array}$ & \\
\hline $\begin{array}{c}\text { Sintomi } \\
\text { muscolo-scheletrici }\end{array}$ & & $\begin{array}{l}1.8 \text { parete } \\
\text { toracica }\end{array}$ & & & & & \\
\hline $\begin{array}{c}\text { Sintomi gastrointestinali } \\
\text { Tratto urinario e malattie } \\
\text { sessuali }\end{array}$ & & 1.7 addominale* & & \begin{tabular}{|c|}
5.7 enteriti non \\
infettive e coliti, \\
4.2 gastriti e \\
gastroduodeniti
\end{tabular} & $\begin{array}{c}43 \\
40 \text { urinarie e ses- } \\
\text { suali }\end{array}$ & 418 & \\
\hline Sintomi psicologici & & & & & 40 & 406 & \\
\hline Eczema & & & & & 39 & 378 & \\
\hline Sintomi respiratori & & & & & 29 & 304 & \\
\hline Infezioni & & $\begin{array}{c}1.2 \text { infiammazio- } \\
\text { ni artic. }\end{array}$ & & & 51 & & \\
\hline \multicolumn{8}{|l|}{ Malattie del sangue } \\
\hline Tumori & 4 solo maligni & 3.5 neuroma & & & & & 3 Solo maligni \\
\hline $\begin{array}{l}\text { Dolore lombare e sintomi } \\
\text { MSK, Altro dolore cronico } \\
\text { non definito }\end{array}$ & $\begin{array}{l}19 \text { non definito } \\
65 \text { malattie } \\
\text { croniche } \\
32 \text { malessere }\end{array}$ & $\begin{array}{l}25.7 \text { dolore } \\
\text { meccanico }\end{array}$ & & & $\begin{array}{c}50 \text { msk } \\
32 \text { altro } \\
\text { non definito }\end{array}$ & $\begin{array}{l}51.6 \text { dolori } \\
\text { muscolo- } \\
\text { scheletrici }\end{array}$ & 4 scoliosi \\
\hline Altro (tendiniti, epicondiliti) & & $12: 3$ & & & & & \\
\hline $\begin{array}{c}\text { Sindrome regionale } \\
\text { miofasciale }\end{array}$ & & $5: 4$ & & & & & \\
\hline Dolore meccanico del collo & & $\begin{array}{c}4.3+2 \text { cervicale } \\
*\end{array}$ & & 7.8 cervicalgia & & & $\begin{array}{l}2 \text { Torcicollo, rigi- } \\
\text { dità nucale }\end{array}$ \\
\hline
\end{tabular}

Tabella 2: Diagnosi cliniche correlate al dolore cronico e Prevalenza (P\%) - Parte A 


\begin{tabular}{|c|c|c|c|c|c|c|c|}
\hline AUTORI & $\begin{array}{l}\text { Rustøen } \\
\text { et al., } 2004\end{array}$ & $\begin{array}{l}\text { Mailis-Gagnon et al., } \\
2007\end{array}$ & $\begin{array}{l}\text { Breivik et al, } \\
2006\end{array}$ & $\begin{array}{l}\text { Koleva } \\
\text { et al., } 2004\end{array}$ & $\begin{array}{l}\text { Eriksen } \\
\text { et al., } 2003\end{array}$ & $\begin{array}{l}\text { Siøgren et al } \\
2009\end{array}$ & $\begin{array}{l}\text { Azevedo et al } \\
2012\end{array}$ \\
\hline $\begin{array}{c}\text { DIAGNOSI CLINICHE } \\
\text { o SINTOMI }\end{array}$ & $\mathrm{P}(\%)$ & $\mathrm{P}(\%)$ & $\mathrm{P}(\%)$ & $\mathrm{P}(\%)$ & $\mathrm{P}(\%)$ & $\mathrm{P}(\%)$ & $\mathrm{P}(\%)$ \\
\hline $\begin{array}{c}\text { Sindrome dolorosa } \mathrm{C} \text {. } \\
\text { regionale }\end{array}$ & & $14: 2$ & & & & & \\
\hline Danno nervoso periferico & & $\begin{array}{c}6.6+2 \text { plesso } \\
\text { brachiale }\end{array}$ & & & & & \\
\hline Danno nervo spinale & & $6: 6$ & & & & & 9 Non specificato \\
\hline Radicolopatia lombare & & $\begin{array}{c}4.8+2 \text { stenosi } \\
\text { spinale }\end{array}$ & & & & & \\
\hline $\begin{array}{l}\text { Dolore neuropatico, } \\
\text { Neuralgia posterpetica e } \\
\text { neuropatia diabetica }\end{array}$ & 2 stroke & $\begin{array}{c}4.8+6.2 \text { neuropati- } \\
\text { co }+4.5 \text { arto fanta- } \\
\text { sma }+4.1 \text { mieolo- } \\
\text { patia, } 3.8 \text { post } \\
\text { stoke, } 7 \text { altro (polio, } \\
\text { Parkinson) }\end{array}$ & & $3: 7$ & & & $\begin{array}{l}1 \text { Solo neuropa- } \\
\text { tia diabetica }\end{array}$ \\
\hline Disordini intervertebrali & & & $\begin{array}{c}15 \text { Ernie discali o } \\
\text { protrusioni }\end{array}$ & & & & 21 \\
\hline Interventi chirurgici & & 2 post toracotomia* & & & & & 6 \\
\hline Sciatalgia & & & & $8: 7$ & & & 4 \\
\hline
\end{tabular}

Tabella 2: Diagnosi cliniche correlate al dolore cronico e Prevalenza (P\%)- Parte B NOTE:

1) Le informazioni relative alla prevalenza sono state descritte dall'autore e classificate percentualmente per Dolore Neuropatico e Sindromi Muscoloscheletrici, per cui il totale non potrà essere il 100\%. I dati contrassegnati da asterisco trattasi di dolore neuropatico $(*)$.

2) Le percentuali di prevalenza sono descritti contemporaneamente in termini sintomatologici e di lunghe malattie, rispetto a varie caratteristiche.

3) Le percentuali di prevalenza sono descritti contemporaneamente in termini sintomatologici e di lunghe malattie, rispetto a varie caratteristiche.

ancora realizzati in strutture sanitarie (Koleva et al., 2005; Malilis-Gagnon et al., 2007), ma non chiaramente nei centri di terapia del dolore.

\section{c) Cause e diagnosi cliniche correlate al dolore cronico e prevalenza.}

Al fine di descrivere le diagnosi emerse dagli studi analizzati si prega di fare riferimento alla Tabella 2 e 3Note esplicative: si potrà notare come in alcuni casi non si parla di diagnosi cliniche vere e proprie ma di sintomatologia dolorosa (dolore lombosacrale, dolore toracico ect..). Inoltre sono descritti i dati percentuali in termini di prevalenza $(\mathrm{P})$, la localizzazione del dolore (apparato per apparato), natura del dolore (neuropatico, viscerale e misto). Queste caratteristiche descritte fanno emergere come il dolore cronico benigno è una condizione clinica caratterizzata da una elevata complessità per la preesistenza molto spesso di pluripatologie che coesistono contemporaneamente e richiedono pertanto trattamenti altrettanto complessi.

\section{Caratteristiche socio-demografiche della popolazione con dolore cronico.}

Nello studio di Rustøen et al, (2004) le caratteristiche socio-demografiche studiate sono il livello di scolarità (distinti per scuola primaria 19\%, e secondaria $44 \%$, scolarità università inferiore a 4 anni $20 \%$ e superiore a 4 anni $17 \%$ ), lo stato civile (sposati coabitanti $58 \%$, non sposati $29 \%$, divorziati/separati $8 \%$, vedovi $5 \%$ ), la co-residenza (sposi $72 \%$, bambini $4 \%$, soli $16 \%$, famiglia/fratelli $4 \%$, altro 4\%), l'attività professionale (occupati 69\%, casalinghe $3 \%$, disoccupati $2 \%$, pensionati $21 \%$, studenti/in servizio militare 5\%). Una larga proporzione di non sposati e occupati non soffrono di dolore cronico. Il dolore sembra essere prevalente nel sesso femminile (55\%), nella popolazione anziana pensionata (35\%), divorziati o separati $(10 \%)$ e quelli con bassa scolarità $(28 \%)$.

Nello studio di Malilis-Gagnon et al., (2007) le variabili studiate sono lo stato civile (risultati: $20 \%$ single, $58 \%$ sposati, $5 \%$ relazioni legalizzate, $11 \%$ separati/divorziati, $5.6 \%$ vedovi; in questa ultima categoria il rapporto femmina-maschi è di 3:1, rispetto alle altre che si dimostrano pressoché simili), l'occupazione (solo il 19.76\% degli intervistati era occupata al momento dell'intervista, che contrasta con un $70.2 \%$ che erano occupati all'inizio dei problemi relativi al dolore; il $13.8 \%$ erano in pensione, inoltre il tasso di disoccupati è aumentato da $16.9 \%$ al momento della comparsa del dolore a $65.4 \%$ al momento della consultazione clinica), la scolarità (circa il $44 \%$ dei soggetti aveva completato le scuola superiore, un altro $20.5 \%$ ha ottenuto un titolo universitario, il $25 \%$ ha frequentato o completato l'università). Nello studio descritto di Eriksen at al., (2003) le variabili studiate sono: lo stato civile (risultati: sposati 19\%, divorziati $27 \%$, vedovi $31 \%$, single $14 \%$, conviventi $36.7 \%$ ), la scolarità (la prevalenza era del 29\% rispetto alle persone con meno di 10 anni di scolarità; i soggetti con maggiore scolarità di 13 anni, avevano un tasso del 14\%), l'occupazione (il $21 \%$ svolgono attività lavorativa caratterizzata da elevato sforzo fisico rispetto al $12 \%$ di coloro che svolgono lavori sedentari).

Nello studio di Siøgren et. al., (2009) le variabili studiate sono: lo stato civile (risultati: sposati $21 \%$, conviventi $17.8 \%$, separati/divorziati/vedovi $29.3 \%$, single $13.3 \%$ ), la scolarità (inferiore a 10 anni, 29.5\%, 11-12 anni $24.9 \%, 13-14$ anni $17.9 \%, \geq 15$ anni $11.8 \%$ ). Esiste una correlazione positiva tra elevata prevalenza e lo stato 
civile separati/divorziati/vedovi, bassa scolarità e peso eccessivo, in linea con un precedente studio (Eriksen et al., 2003). La prevalenza, dopo aggiustamento per sesso ed età, viene stimata del $20.0 \%$. Lo studio di Koleva et al., (2005) non descrive invece correlazioni con le variabili socio-demografiche. Lo studio multicentrico di Breivick at al., (2006) descrive tra le variabili socio-demografiche l'occupazione (risultati: full-time 31\%, parttime $13 \%, 34 \%$ pensionati, $22 \%$ disoccupati). Uno su 4 dei soggetti occupati riferisce che il dolore ha un impatto sulla propria attività. La media di giorni persi di lavoro a causa del proprio dolore, in tutti Paesi studiati è in media stimato di 7.8 giorni.. Il 55\% non ha perso giorni di lavoro, l'11\% ha perso da 1 a 3 giorni, il $12 \%$ ha perso da 4 a 9 giorni, il $9 \%$ ha perso da 10 a 15 giorni, il $13 \%$ ha peso almeno 16 giorni. La Finlandia sembra quella che ha perso più giorni di lavoro, in media quasi 20 giorni, mentre la Francia solo 5 giorni; l'Italia in media ha perso 7.6 giorni. Lo studio di Azevedo et al., (2012) mostra come il dolore è prevalente nel sesso femminile $(52,3 \%)$ rispetto ai maschi $(47,7 \%)$. La prevalenza ha un trend elevato nella fascia di età tra 18-24 anni (12,9\%), che tende a ridursi con l'aumentare dell'età fino alla fascia di età compresa tra $70-74(5,5 \%)$, per poi aumentare $(8,4 \%)$. Rispetto allo stato civile emerge che la prevalenza si distribuisce come segue: single $24,8 \%$, sposati o uniti civilmente $61,9 \%$, divorziati-separati $4,2 \%$, vedovi $9,1 \%$. L'indagine mostra una significativa associazione tra dolore e sesso femminile, età avanzata, basso livello di istruzione, tra i soggetti disoccupati e pensionati.

\section{DISCUSSIONE}

L'analisi di questi sette articoli evidenzia come le metodologie utilizzate per determinare la prevalenza del dolore cronico siano diverse, seppure quella telefonica o per intervista siano le più utilizzate seppure appartengano alle metodologie utilizzate nella ricerca qualitativa. Sembra che si tratti di strumenti non sottoposti preventivamente a valutazione della validità ed affidabilità. Mentre la validità si riferisce al fatto che uno strumento di valutazione misura appunto accuratamente ciò per cui è stato creato, l'affidabilità è una misura statistica che misura la riproducibilità di uno strumento (LoBiondo-Wood \& Singh, 2013). Per quanto riguarda le altre tecniche di intervista, appunto quella telefonica, seppure mette in evidenza un elevato tasso di risposta (Bassols et al., 1999; Blyth et al., 2001; Catala et al., 2002; $\mathrm{Ng}$ et al., 2002), può minare la rappresentatività, sia perché alcuni non possono essere presenti nella lista telefonica, o perché altri non erano a

\begin{tabular}{|c|c|}
\hline Articoli & Note aggiuntive esplicative \\
\hline $\begin{array}{l}\text { Breivik et al., } \\
2006\end{array}$ & $\begin{array}{l}\text { Osteoartriti and artrite reumatoide si presenta con un } 38 \%-48 \% \text { in UK, Irlanda, Italia, Spagna, Norvegia, Belgio; } 8-10 \% \text { in Finlandia e } \\
\text { Israele, } 17-31 \% \text { Austria, Germania, Francia. Erniazione o deterioramento del disco intervertebrale sono comuni in Austria, Svizzera, } \\
\text { Belgio (25\%), mentre in Norvegia, Svezia, Finlandia and Danimarca per il 6-14\%; cefalea ed emicrania appaiono essere molto comuni } \\
\text { in Germania, Francia, Polonia, Austria, Svizzera ed in altri Paesi }\end{array}$ \\
\hline $\begin{array}{l}\text { Koleva et al., } \\
2004\end{array}$ & $\begin{array}{l}\text { Molte patologie sono state osservate rapportandole tra femmina e maschio: cefalea (3.3:1), enteriti non infettive } \& \text { coliti }(2.3: 1) \text {, } \\
\text { malattie dell'esofago, stomaco e duodeno }(1.7: 1) \text { mialgia non specificata \& miositi }(2.1: 1) \text {. II dato femminile prevale con un rapporto } \\
\text { 1.8:1 su quello maschile. I dati non si rivolgono esclusivamente al dolore cronico ma anche a quello acuto (P } 47.2 \%) \text {. Lo studio è rea- } \\
\text { lizzato tra la popolazione che afferisce alla medicina generale. }\end{array}$ \\
\hline $\begin{array}{l}\text { Eriksen et al., } \\
\quad 2003\end{array}$ & $\begin{array}{l}\text { Realizzando una regressione logistica si mostra una associazione tra dolore cronico e storia di cancro. diagnosi di tumore presente } 33.1 \% \\
\left.\left(\mathrm{OR}^{1} 1.54, \mathrm{Cl} 0.86-2.78\right) \text {, diagnosi precoce di cancro } 31.0 \% \text { (OR } 1.39, \mathrm{Cl} 1.02-1.90\right) \text {, mai diagnosticato patologia neoplastica } 20.2 \%(\mathrm{OR} 1) \text {. }\end{array}$ \\
\hline $\begin{array}{l}\text { Mailis-Gagnon et } \\
\quad \text { al., } 2007\end{array}$ & $\begin{array}{l}\text { È stato utilizzato il DSM-IV-TR per la classificazione dei disturbi, metodo problematico e controverso. include i soggetti in } 3 \text { gruppi: } \\
\text { Gruppo I (pazienti con dolore associato a condizioni mediche generali), Gruppo II (pazienti con dolore associato a fattori psicologici } \\
\text { insieme a condizioni mediche generali), Gruppo III (pazienti con dolore associato a fattori psicologici). Rispetto ai gruppi predefiniti } \\
\text { (in questo caso Gruppo I), come negli uomini prevalgono condizioni elevati livelli di cliniche o dimostrabili da patologie organiche, } \\
\text { mentre le femmine facenti parte del Gruppo III, caratterizzati da significativi fattori psicologici coinvolti nello studio. Si fa presente } \\
\text { che lo studio individua aree patologiche e non un unico dato, per cui il dato di Prevalenza cumulativo descritto in tabella } 2 \text { non potrà } \\
\text { essere il } 100 \% \text {. I risultati in termini di prevalenza vengono cosi descritti: dolore neuropatico } 33.1 \% \text { maschio e } 27.5 \text { femmina; dolore } \\
\text { muscolo-scheletrico } 35.7 \% \text { maschio e } 45 \% \text { femmina; dolore viscerale } 2.5 \% \text { maschio e } 3.8 \% \text { femmina; sindrome da dolore toracico e } \\
\text { failed back surgery syndrome (FBSS) } 6.2 \text { maschio e } 4.42 \text { femmina; dolore misto (muscolo scheletrico e neuropatico) } 15.5 \% \text { maschio e } \\
10.38 \text { femmina; altro } 3 \% \text { maschio e } 3.6 \text { femmina. }\end{array}$ \\
\hline $\begin{array}{l}\text { Azevedo et al., } \\
2012\end{array}$ & $\begin{array}{c}\text { Realizzando una regressione logistica si mostra una associazione tra dolore cronico e variabili socio demografiche emerge una } P \\
\text { value (vedi nota) significativa }(<0.001) \text { OR }[95 \% \mathrm{IC}] \text { relative a sesso, età, occupazione, livello di scolarità. }\end{array}$ \\
\hline $\begin{array}{c}\text { Siøgren et al } \\
2009\end{array}$ & $\begin{array}{c}\text { Questo articolo è un aggiornamento di dati relativi al'epidemiologia del dolore cronico benigno già realizzato nel } 2000 \text { e nel } 2005 \\
\text { nello stesso Paese (Danimarca). Lo studio da una regressione logistica mostra una associazione tra dolore cronico e storia di patolo- } \\
\text { gia neoplastica, seppure lo studio si focalizza su quello benigno. Inoltre sembra ci sia una interessante correlazione tra dolore cronico } \\
\text { e essere single, basso livello di educazione-scolarità. La correlazione positiva tra aumenti dell'età e dato di prevalenza può essere } \\
\text { controversa, legato allo stato di percezione nocicettiva del paziente . }\end{array}$ \\
\hline $\begin{array}{l}\text { Rustøen et al., } \\
2004\end{array}$ & $\begin{array}{c}\text { I dati descritti risultano statisticamente significativi allo } 0.05 \text { utilizzando il post-hoc tests tra la differenza delle proporzioni. Non è } \\
\text { noto come acuni pazienti non attribuiscano il loro dolore ad una patologia nota (studio sulla popolazione generale). Oltre all'età, la } \\
\text { bassa scolarità sembra fare riportare dolore frequentemente. Non è stato usato un questionario validato per la misurazione degli } \\
\text { items di interesse, oltre ad altri oramai noti in letteratura (BPI). La maggior parte della popolazione che ha partecipato trattasi di } \\
\text { donne sposate, con possibile bias di campionamento. }\end{array}$ \\
\hline
\end{tabular}

1 Aggiustata per sesso ed età, anche nei casi successivi descritti.

Tabella 3: Approfondimenti risultati 
casa al momento della chiamata. Non è detto che tutti abbiano un telefono a domicilio, visto il diffuso uso di telefonia mobile. Inoltre l'affidabilità delle informazioni riferite da chi risponde al telefono possono essere meno accurate e con conseguente potenziamento dell'errore sistematico (bias di informazione relativo sia a quello di recall che dell'intervistatore) (Faggiano et al., 2005) . Acquisire informazioni cliniche dalla documentazione sanitaria acquista maggiore credibilità, in quanto le valutazioni e rilevazioni da parte del medico sono maggiormente rigorose e credibili rispetto ai questionari postali autocompilati (Verhaak et al, 1998) o di quelli inviati e compilati tramite la via mail. Inoltre le controversie relative alla identificazione temporale per definire il dolore cronico da quello acuto dà dubbie interpretazioni. Il dolore cronico è stato riconosciuto come quel dolore che persiste oltre il normale tempo di guarigione (Bonica, 1953), identificando tre tempi con le rispettive categorie: inferiore a 1 mese, da 1 a 6 mesi, superiore a 6 mesi (IASP Subcommittee on Taxonomy, 1994). L'utilizzo di una comune e multidimensionale definizione di dolore cronico ha una forte influenza sui risultati di prevalenza (Verhaak et al., 1999); inoltre negli studi emerge come il concetto di dolore benigno non ne viene fatto uso esplicito (IASP, 2003). Il dolore cronico non è più sintomo ma è malattia vera e propria (Varrassi et al., 2011), e data la complessità della natura del dolore non oncologico, esso non permette una misurazione in termini di prevalenza circostanziata come una semplice condizione fisica, bensì richiede un approccio multidimensionale che coinvolge tutte le caratteristiche che lo definiscono: intensità, strategie di coping ed una esperienza personale in risposta al dolore, localizzazione, caratteristiche temporali, valutazione efficace (Von Korff et al., 1990; IASP, 2005). Pertanto i dati relativi alla prevalenza saranno caratterizzati da una certa variabilità, legata anche al target dei pazienti studiati (popolazione rurale, popolazione istituzionalizzata, popolazione afferente ai centri di terapia del dolore). Il dato di prevalenza è maggiormente rappresentativo nelle femmine ed aumenta con l'aumentare dell'età (Andersson, 2004), anche se in letteratura viene descritto come l'aumento dell'età può ridurre la capacità cognitiva di riportare il dolore (Fisher et al., 2006). L'associazione tra prevalenza, età e dolore è controversa. Basti pensare che molti anziani possono avere una diminuzione dell'attività di nocicezione e di accettazione del dolore come “compagno" dell'aumento dell'età, per paura di essere respinti o essere troppo dipendenti dagli altri o dai farmaci analgesici (Yates et al., 1995). Dal punto di vista socio demografico, la scolarità è un forte indicatore dello stato socioeconomico (Elliott et al., 1999; Eriksen et al., 2003, Wong et al., 2011), motivo per cui la bassa scolarità corrisponde ad un reddito più basso ed a potenziali peggiore qualità di vita. Inoltre pensionati e disoccupati sono le fasce sociali più deboli con maggiore rischio di dolore cronico (Elliott et al., 1999; Eriksen et al., 2003; Yu et al., 2006; Wong et al., 2011).

Dal punto di vista diagnostico spesso si tratta di sintomatologia dolorosa e non è nota la diagnosi vera e propria come descritto negli studi analizzati. I limiti di questa revisione sono legati al fatto che studia una piccola parte della grande produzione epidemiologica presente in letteratura, escludendo le classi pediatriche e geriatriche, e tutti gli articoli finalizzati a dare informazioni sulle monopatologie che caratterizzano il dolore cronico benigno. Lanalisi narrativa degli articoli non descrive volutamente i trattamenti farmacologici e complementari e l'impatto che il dolore cronico o ha sulle attività di vita quotidiana, che meritano un approfondimento specifico.

\section{CONCLUSIONI}

Questa revisione, nonostante descriva un limitato numero di studi, fa emergere come il fenomeno del dolore cronico non oncologico sia ampio tra le popolazioni studiate in molti paesi Europei e non. Sono descritte innumerevoli condizioni cliniche di natura benigna e sintomi che lo caratterizzano, rendendo questo problema di salute così diffuso anche ancor più complesso e meno noto tra gli operatori sanitari che non se ne occupano specificatamente. La sua natura benigna (non maligna) potrebbe conferirgli un'attenzione, da parte del personale sanitario e da parte delle istituzioni, diremmo epidermica, in quanto non viene immediatamente correlata all'exitus. Il legislatore però, anche se solo negli ultimi anni (Legge n.38/2010), ha mostrato maggiore sensibilità ad un problema di salute apparentemente ovvio, a tutt'oggi rischia di essere sottovalutato e sottotrattato, benchè siano ormai note le sequele in termini di morbilità e disabilità rispetto all'alterazione delle attività di vita $(\mathrm{ADL})$ non descritti volutamente in questa revisione. Il dolore cronico inoltre è spesso associato ad altre comorbidità , quali la depressione ed al potenziale rischio di suicidio, che necessitano di trattamenti specifici, globali e multiprofessionali, dove la componente affettiva emozionale prevale su quella somatica, e che impone pertanto l'intervento di operatori sanitari dotati di elevata competenza professionale specifica, dove spesso le cure primarie rimangono impotenti. Un fenomeno così importante e vasto dal punto di vista epidemiologico, seppure descritto ampiamente in letteratura, non trova però rispettivi numerosi studi sulla popolazione italiana. Comprendere l'ampiezza reale del fenomeno dal punto di vista epidemiologico nel nostro Paese garantirebbe una maggiore consapevolezza da parte del legislatore che si occupa anche di programmazione delle politiche sanitarie e dei servizi (quali e quanti) necessari, da distribuire sul territorio nazionale, sia ospedaliero (hub) e distrettuale (spoke) al fine di garantire una efficace risposta da parte di una rete in grado di 
soddisfare la richiesta di cura da parte della popolazione affetta da dolore cronico (IASP, 1994). Infatti sembra in Italia siano presenti un numero esiguo di centri di terapia del dolore (Notaro, 2012). Infine gli autori intravedono nella figura infermieristica un ruolo chiave rispetto alle nuove prospettive formative e accademiche, in merito alla potenziale possibilità di impiegarla, con una competenza specifica avanzata, nei confronti delle strategie preventive necessarie per ridurre i casi di cronicizzazione del dolore acuto e nella gestione globale della persona affetta da dolore cronico.

\section{BIBLIOGRAFIA}

Abu-Saad, H. (2010). Chronic Pain: a review. Lebanese Medical Journal, 58 (1), 21-27.

Andersson, H.I. (2004). The course of non-malignant chronic pain: a 12-year follow-up of a cohort from the general population. European Journal of Pain, Feb; 8 (1), 47-53.

Azevedo, L.F., Costa-Pereira, A., Mendonça, L., Dias, C.C., \& Castro-Lopes, J.M. (2012) Epidemiology of chronic pain: a population-based nationwide study on its prevalence, characteristics and associated disability in Portugal. The Journal of Pain, 13 (8), 773-783.

Bassols, A., Bosch, F., Capillo, M., Caňellas, M., Baňos, J.E. (1999). An epidemiologoical comparison of pain complaints in the general population of Catalonia (Spain). Pain, 83:9-16.

Bekkering, G.E., Bala, M.M., Reid, K., Kellen, E., Harker, J., Riemsma, R., Huygen F.J.P.M., Kleijnen, J (2011). Epidemiology of chronic pain and its teatment in the Netherlands. The Jpurnal of Medicine, March 69 (3), 141-152.

Blyth, F.M., March, L.M., Brnabic, A.J., Jorm, L.R., Williamson, M., Cousins, M.J. (2001). Chronic pain in Australia: a prevalence study. Pain, 89 (2-3), 127-34.

Blyth, F.M., March, L.M., Brnabic, A.J.M., Cousins, M.J. (2004). Chronic pain and frequent use of healthcare. Pain, 111 (1-2), 51-8.

Bonica's Trattamento del dolore, in Loeser, J.D.(2003). Antonio Delfino Editore, Roma.

Breivik, H., Collett, B., Ventafridda, V., Cohen, R., Gallacher, D. (2006). Survey of chronic pain in Europe: prevalence, impact on daily life, and treatment, European Journal of Pain, 10 (4), 287-333.

Catala, E., Reig, E., Artes, M., Aliaga, L., Lopez, JS, Segu, J.L. (2002). Prevalence of pain in the Spanish population: telephone survey in 5000 homes. European Journal of Pain, 6, 133-40.

Elliott, A.M., Smith, B.H., Penny, K.I., Smith, W.C., Chambers, W.A. (1999). The epidemiology of chronic pain in the community. Lancet, 354, 1248-52

Eriksen, J., Jensen, M.K., Siøgren, P., Ekholm, O., Rasmussen, N.K. (2003) Epidemiology of chronic non-malignant in Denmark. Pain, 106, 221-228.

Eriksen, J., Siogren, P., Eklom, O., Rasmussen, N.K. (2004). Healthcare utilization among individuals reporting longterm pain: an epidemiological study based Danish National Health Surveys. European Journal of Pain, 8(6), 517-23.

European Federation of the IASP Chapter (EFIC). (2011). Cost of chronic pain, http://www.efic.org/chronic pain.html, accessed 8 February.

Faggiano, F., Donato, F., Barbone, F. (2005). Manuale di Epidemiologia per la Sanità Pubblica. Centro Scientifico Editore, SItI.

Fisher, S.E., Burgio, L.D., Thorn, B.E., Hardin, J.M. (2006)
Obtaining self-report data from cognitively impaired elders: methodological issues and clinical implications for nursing home pain assessment. Gerontologist, 46, 81-8.

Gallagher, R.M. (1999). Primary care and pain medicine. A community solution to the public health problem of chronic pain. Medical Clinics of North America, 83, 555-83.

International Association of Study of Pain. (1999). Epidemiology of pain. IASP Press Seattle.

International Association of Study of Pain. (2003). Pain Clinical Update, June vol. XI n.2

International Association of Study of Pain. (2005). Core Curriculum for professional education in pain. IASP Press Seattle.

Joint Commission International. (2010). Approaches to pain management, an essential guide for clinical leaders, Second edition.

Koleva, D., Krulichova, I., Bertolini, G., Caimi, V., Garattini, L. (2005). Pain in primary care: an Italian survey. European Journal of Public Health, 15 (5), 475-479.

LoBiondo-Wood, G., \& Haber, J. (2013). Nursing Research in Canada. Methods, Critical Appraisal and Utilization. Elsevier, $3^{\circ}$ Edition.

Malilis-Gagnon., A., Yesgneswaran, B., Lakha, S.F., Nicholson, K., Steiman, A., Ng, D., Papagapiou, M., Umana, M., Cohodarevic, T., Zurowski, M. (2007). Pain characteristic and demographics of patients attending a university-affiliated pain clinic in Toronto, Ontario, Pain Research \& Management, 12 (2), 93-99.

Niv, D., \& Devor, M. (2004). Chronic pain is a disease in its own right. Pain Practice, 4, 179-181.

Ng, K.F., Ysui, S.L., Chan, W.S. (2002). Prevalence of common chronic pain in Hong Kong adults. Clinical Journal of Pain, 18, 275-81.

Notaro, P. (2012). Dolore cronico, dolore inutile. Strutture di Terapia del Dolore in Italia. Perché un aggiornamento del Libro Bianco. NOPAIN Onlus

Rustøen, T., Wahl, A.K., Hanestad, B.R., Lerdal, A., Paul, S. Miaskowski, C. (2004). Prevalence and characterist of chronic pain in the general Norwegian population. European Journal of Pain, 555-565.

Siøgren, P., Ekholm, O., Peuckmann, V., Grønbæk, M. (2009). Epidemiology of Chronic pain in Demmark. European Journal of Pain, 13 (3), 287-292.

Sullivan, M.D., Turner, J.A., Romano, J. (1991). Chronic pain in primary care. Identification and management of psychosocial factors. The Journal of Family Practice, 32, 193-9.

Varrassi, G., Collett, B., Morlion, B., Kalso, E., Nicolaou, A., Dickenson, A., Pergolizzi, J., Schäfer, M., MüllerSchwefe, G. Proceedings of the CHANGE PAIN Expert Summit in Rome, June 2010. Current Medical Research \& Opinion. 2011 Oct;27(10):2061-2. Epub 2011 Sep 20.

Verhaak, P.F.M., Kerssens, J.J., Sorbi, M.J., Bensing, J.M. (1998). Prevalence of chronic benign pain disorder among adults: a review of the literature. Pain, 77 (3), 231239.

Von Korff, M., Dworkin, S.F., Le Resche, L. (1990). Graded chronic pain status: an epidemiologic evaluation. Pain, 40, 279-291.

Widerström-Noga, E.G. (2009). Pain: A multidimensional problem of national priority. The Journal of Rehabilitation Research and Development, 46(1), vii-ix.

Wong ,W.S., Fielding, R. (2011). Prevalence and characteristics of chronic pain in the general population of Hong Kong. Journal of Pain, 12, 236-245,

Yates, P., Dewar, A., Fentiman, B. (1995). Pain: the views of elderly people living in long-term residential care settings. Journal of Advanced Nursing, Apr; 21 (4), 667-74.

Yu, H.Y., Tang, F.I., Kuo, B.I., Yu, S. (2006). Prevalence, interference, and risk factors for chronic pain among Taiwanese community older people. Pain Management Nursing, 7, 2-11. 